\title{
Dynamics of cinacalcet use and biochemical control in hemodialysis patients: a retrospective New-user cohort design
}

\author{
B. Diane Reams ${ }^{1}$, Paul J. Dluzniewski ${ }^{2}$, Thy P. Do², Susan V. Yue ${ }^{2}$, Brian D. Bradbury ${ }^{2}$, Abhijit V. Kshirsagar ${ }^{3}$ \\ and M. Alan Brookhart ${ }^{4 *}$
}

\begin{abstract}
Background: Cinacalcet is used to treat secondary hyperparathyroidism among hemodialysis patients. Large-scale epidemiologic studies describing patterns of cinacalcet use, effects on parathyroid hormone (PTH), calcium, and phosphorous levels, and predictors of discontinuation have not been previously reported.

Methods: This retrospective cohort study used a clinical database of a large U.S. dialysis provider (2007-2010) merged with administrative data from the United States Renal Data System. Among new users of cinacalcet with Medicare coverage, trends in PTH, calcium, and phosphorus were measured in 30-day intervals following cinacalcet initiation.

Results: Seventeen thousand seven hundred sixty-three eligible initiators contributed 111,047 30-day follow-up intervals. Of these, $56 \%$ discontinued cinacalcet by month 4 . Of those discontinuing, $76.3 \%$ reinitiated. Mean values of PTH, calcium, and phosphorus decreased to recommended levels within 4 months following initiation. Proximal PTH levels $<150 \mathrm{pg} / \mathrm{mL}$ were associated with discontinuation: $\mathrm{HR}=1.23$ (95\% Cl: 1.12, 1.36), whereas low calcium $(<7.5 \mathrm{mg} / \mathrm{dL})$ was suggestive of an association, $\mathrm{HR}=1.09(95 \% \mathrm{Cl} 0.91,1.32)$. Being in the Part $\mathrm{D}$ gap period increased discontinuation risk: $\mathrm{HR}=1.09$ (95\% Cl: 1.03, 1.16). Low-income subsidy status decreased discontinuation risk: $\mathrm{HR}=0.77(95 \% \mathrm{Cl} 0.69,0.86)$. Predictors of reinitiation included low-income subsidy, $\mathrm{HR}=1.32$ (95\% Cl 1.22, 1.43); higher albumin level, $\mathrm{HR}=1.23(95 \% \mathrm{Cl} 1.10,1.36)$ and higher calcium level, $\mathrm{HR}=1.26(95 \% \mathrm{Cl}$ $1.19,1.33)$.
\end{abstract}

Conclusions: Substantial and expected declines in laboratory values occurred following cinacalcet initiation. Early discontinuation and reinitiation of cinacalcet were common and may have occurred for clinical and economic reasons.

Keywords: Cinacalcet, Secondary hyperparathyroidism, Hemodialysis

\section{Background}

Secondary hyperparathyroidism (SHPT) among patients with chronic kidney disease (CKD) results from decreased active vitamin $\mathrm{D}$ and is manifested by low serum calcium and elevated levels of phosphorus and parathyroid hormone (PTH) [1, 2]. Treatment options to control CKD-MBD parameters include modulation of calcium and phosphorous balance through dietary intake and dialysis, active vitamin D compounds, and phosphate

\footnotetext{
* Correspondence: abrookhart@unc.edu

${ }^{4}$ Department of Epidemiology, UNC Gillings School of Global Public Health, UNC Chapel Hill, Chapel Hill, NC, USA

Full list of author information is available at the end of the article
}

binders [3]. The Kidney Disease: Improving Global Outcomes (KDIGO) guideline recommendations for the optimal range of PTH is two to nine times the assay's upper limit of normal reference range, with levels not to exceed approximately $600 \mathrm{pg} / \mathrm{mL}$. Practitioners are also recommended to target the assay reference range for calcium and phosphorus [4].

Another therapeutic option is cinacalcet (Sensipar\% Mimpara $^{\circ}$, Amgen Inc., Thousand Oaks, CA), a calcimimetic agent that directly lowers PTH, and subsequently, calcium and phosphorus [5, 6]. However, post-marketing studies in real world settings have found adherence to cinacalcet to be sporadic, which may limit its effectiveness 
[7-9]. Identifying factors associated with discontinuation may help healthcare providers better understand and prevent non-adherence to therapy. Previous studies examining adherence to cinacalcet have been limited by size, the inability to identify time-varying covariates, and inaccuracies in identifying therapy start and stop dates [7, 10-12].

Using detailed clinical, laboratory, and healthcare utilization data from a large cohort of patients receiving hemodialysis, we sought to describe the experience of patients initiating cinacalcet, including the trajectory of biochemical parameters following initiation, as well as factors predicting discontinuation and reinitiation of therapy.

\section{Methods}

\section{Data sources}

Our study used 4 years of data (January 1, 2007 through December 31, 2010) from the United States Renal Data System (USRDS) linked to data from a large dialysis provider. The dialysis provider owns and manages over 1,500 outpatient dialysis facilities located throughout the U.S. in urban, rural, and suburban areas. Their clinical database captures detailed clinical, laboratory, and treatment data on patients receiving care at all of their dialysis units. Data are collected using standardized clinical protocols and electronic record systems. The USRDS is a national data system, funded by the National Institute of Diabetes and Digestive and Kidney Diseases, which collects, analyzes, and distributes information about the treatment of endstage renal disease (ESRD). The USRDS data include data from the Medical Evidence Report Form, Medicare Enrollment database, ESRD Death Notification Form, as well as standard analytic files that contain final action claims for Medicare Parts A, B, and D services [13]. Medicare Parts A through $\mathrm{D}$ are government provided insurance programs for the elderly, disabled, and those with certain medical conditions. Permission to use USRDS data was obtained from The National Institute of Diabetes and Digestive Kidney Diseases. These data were used to obtain information on demographic and comorbid characteristics and health care utilization, such as hospitalizations and outpatient care.

\section{Study design}

Our study used a retrospective new-user cohort design [14]. Patients were eligible to be included in our study if they were 18 years and older, had one year of continuous Medicare Parts A, B, and D coverage during the study period, and had in-center hemodialysis at the large dialysis provider's facility. During a 6-month baseline period, we identified potential confounders and effect modifiers. Among all eligible patients, new cinacalcet users were defined as patients who filled at least one 30-day cinacalcet prescription without use in the prior 6 months (baseline period) based on USRDS Medicare Part D claims files. New users were also required to have received hemodialysis for at least 9 months and had at least 9 dialysis sessions during the last month of the baseline period. Patients receiving a parathyroidectomy during the baseline period or 1st 30-day fill of cinacalcet were excluded. The cohort construction is outlined in Additional file 1: Figure S1.

The start of follow-up was on day 31 following an initial 30-day fill of any dose of cinacalcet. At the end of each 30-day interval, the patient's treatment status was determined. Patients were defined as discontinuing cinacalcet if there was greater than a 30-day gap from their last pill day, calculated from the days supply dispensed. Patients were then followed from discontinuation until first reinitiation of cinacalcet. Because we did not know the exact date of discontinuation or reinitiation in a given interval, the 30-day interval prior to the discontinuation or reinitiation interval was used for the identification of timevarying covariates including laboratory values, cardiovascular and renal risk factors, and medications. The most recent laboratory or covariate information in the 30-day interval prior to discontinuation or reinitiation was documented. If laboratory information was not available in the 30-day interval, the most recent value in the prior 90 days was documented. Study schemata are provided in Additional file 1: Figures S2 and S3. In addition, changes in quintile distributions of PTH, calcium, and phosphorus were examined over time in order to assess how increases and decreases between quintiles predict discontinuation and reinitiation. A sensitivity analysis was performed to determine if reinitiation results were modified when biochemical results from 5,7 , and 14 days prior to the date of the laboratory value most proximal to reinitiation were used to predict reinitiation. This lag time could account for any delays between physician recognition of a laboratory abnormality and a decision to reinitiate cinacalcet.

\section{Study variables \\ Outcomes}

Our outcomes of interest were PTH, calcium, and phosphorus serum laboratory values measured monthly following cinacalcet initiation and discontinuation, as well as those leading up to reinitiation of cinacalcet.

\section{Covariates}

Descriptions and definitions of baseline and time-varying covariates are provided in Additional file 1: Table S1 and Table S2. We identified from Medicare and linked clinical data demographic, laboratory, and clinical variables, as well as comorbidities. Covariates included demographic characteristics (e.g., age, sex, race, Medicaid eligibility, census region, year), clinical characteristics (e.g., cause of ESRD, time on dialysis, body mass index, type of vascular access, number of hospital days), baseline laboratory 
variables (e.g., PTH, calcium, phosphorus), time-varying laboratory variables divided into categories consistent with recognized normal and abnormal values, and several timevarying comorbidity measures. International Classification of Diseases Ninth Revision codes, Current Procedural Terminology codes, and Healthcare Common Procedure Coding System codes were used to identify comorbidities and procedures.

\section{Statistical analysis}

To describe the treatment groups, we report means and frequencies of all covariates within each treatment group and subgroups of interest. Trends in means for each lab value were plotted for the months after initiation and discontinuation. These plots were smoothed and $95 \%$ confidence intervals computed using smoothing splines. For each 30-day interval, we fit a logistic model and estimated the probability of discontinuation or reinitiation given the most recent time-varying covariates in the 90 days prior to the 30-day interval in which discontinuation or reinitiation was thought to have occurred. Although each patient could contribute multiple 30-day intervals to the analytic dataset, we did not adjust for repeated measures since each patient, per our study design, can only discontinue once. Patients were censored administratively on December 31, 2010, and for loss to follow-up, transplant, discontinuation of hemodialysis, death, parathyroidectomy, or loss of Medicare Parts A, B, or D coverage. We also described trends in biochemical parameters following treatment discontinuation and leading up to reinitiation across a range of a priori identified subgroups, including those defined by age, time on dialysis, and race.

This research was determined to be exempt from review by the UNC Institutional Review Board. All statistical analyses were conducted using the R Statistical Software version 3.3 [15] and SAS software, Version 9.3, SAS Institute Inc., Cary, NC, USA.

\section{Results}

We identified 17,763 patients who met our study entry requirements and contributed 111,047 30-day follow-up intervals. Table 1 presents patient characteristics of the primary cohort stratified by gender. All covariates considered can be found in Additional file 1: Table S3. At cinacalcet initiation, the average age was 56.7 years (standard deviation (SD) 14.5 years) and the average time on dialysis was 4.5 years (SD 4.3 years), $49.3 \%$ of the cohort was female, and $53.8 \%$ were African American. Several baseline financial factors were also identified. A history of receiving Medicaid benefits or having low-income subsidy was identified in 68.7 and $83.9 \%$, respectively. Mean PTH calcium, and phosphorus at initiation were $642 \mathrm{pg} / \mathrm{mL}$ (SD $519 \mathrm{pg} / \mathrm{mL}$ ), $9.4 \mathrm{mg} / \mathrm{dL}$ (SD $0.7 \mathrm{mg} / \mathrm{dL}$ ), and $5.9 \mathrm{mg} /$ $\mathrm{dL}$ (SD $1.7 \mathrm{mg} / \mathrm{dL}$ ), respectively.
Trends in the mean serum lab values of PTH, calcium, and phosphorus for the first 12 months following cinacalcet initiation, as well as after discontinuation, are shown in Figs. 1, 2 and 3. Following cinacalcet initiation, the levels of the three lab values fell and stabilized at approximately month 4. Mean PTH levels decreased from a baseline level of $642 \mathrm{pg} / \mathrm{mL}$ (SD $519 \mathrm{pg} / \mathrm{mL}$ ) to levels between 375 and $400 \mathrm{pg} / \mathrm{mL}$ in months 4 through 12 . Mean calcium levels decreased from a baseline level of $9.4 \mathrm{mg} / \mathrm{dL}$ (SD $0.7 \mathrm{mg} / \mathrm{dL}$ ) to approximately $8.9 \mathrm{mg} / \mathrm{dL}$ during the 1st month and remained at levels slightly higher than $8.9 \mathrm{mg} / \mathrm{dL}$ between months 4 through 12 . Of the 111,047 30-day intervals examined, 17,980 intervals $(16.7 \%$ ) had a PTH level less than $150 \mathrm{pg} / \mathrm{mL}$ and $1,934(1.8 \%)$ had a calcium level less than $7.5 \mathrm{mg} / \mathrm{dL}$, Additional file 1: Table S4. Phosphorus levels decreased from a baseline mean level of $5.9 \mathrm{mg} / \mathrm{dL}$ (SD $1.7 \mathrm{mg} / \mathrm{dL}$ ) to between 5.2 and $5.4 \mathrm{mg} / \mathrm{dL}$ in months 4 through 12 . Following discontinuation, mean levels of PTH, calcium, and phosphorus increased and then stabilized at higher mean levels than those achieved while on therapy.

The probability of discontinuation by month 4 was $56 \%$ and by month 12 was $73 \%$. Of those who discontinued $(N=12,521), 76.3 \%(N=9,558)$ reinitiated cinacalcet. The mean time to reinitiation was 4.0 months. Predictors of cinacalcet discontinuation and reinitiation are presented in Table 2. All covariates considered can be found in Additional file 1: Table S5. Baseline PTH, calcium, and phosphorus serum levels were not associated with discontinuation; however, levels most proximal to discontinuation were. Low proximal levels of PTH $(<150 \mathrm{pg} / \mathrm{mL})$ were associated with discontinuation, HR 1.23 (95\% CI $1.12,1.36)$. There was a slight association between calcium $(<7.5 \mathrm{mg} / \mathrm{dL})$ and discontinuation, HR 1.09 (95 \% CI 0.91, 1.32). Increasing levels of $\mathrm{PTH}$ and calcium over time, based on changes in quintile distributions, were also associated with discontinuation, HR 1.15 (95\% CI 1.07, 1.23) and HR 1.24 (95\% CI 1.16, 1.32), respectively. The dose of cinacalcet most proximal to discontinuation also predicted discontinuation in this population. Compared to $30 \mathrm{mg}$, a recent dose of $60 \mathrm{mg}$ of cinacalcet slightly predicted discontinuation, HR 1.07 (95 \% CI 1.00, 1.15), while a recent dose of $90 \mathrm{mg}$ compared to $30 \mathrm{mg}$ had a stronger association, HR 1.15 (95 \% CI 1.03, 1.29). Other factors associated with discontinuation included increasing copay, in the follow-up period, HR 1.04 (95\% CI 1.02, 1.07); time spent in the hospital, HR 2.02 (95\% CI 1.84, 2.22); being in the Medicare Part D gap period, HR 1.09 (95 \% CI 1.03, 1.16); and a diagnosis of stroke during follow-up, HR 1.30 (95\% CI 1.06, 1.60). Nausea, vomiting, and diarrhea were not common in our study given the limitation of ICD-9 diagnosis codes to identify these outcomes, $N=1,30830$ day intervals $(1.2 \%)$ and were only slightly predictive of discontinuation, HR 1.09 (95 \% CI 0.91, 1.32). Proximal 
Table 1 Baseline characteristics overall and by gender

\begin{tabular}{|c|c|c|c|}
\hline Characteristic $^{a}$ & Total & Female & Male \\
\hline \multicolumn{4}{|l|}{ Demographics } \\
\hline Patients, $N$ & 17,763 & 8,764 & 8,999 \\
\hline Age, mean (SD), years ${ }^{b}$ & $56.7(14.5)$ & $59.1(14.8)$ & $54.4(13.9)$ \\
\hline Time on dialysis, mean (SD), years ${ }^{\mathrm{b}}$ & $4.5(4.3)$ & $4.4(4.1)$ & $4.7(4.4)$ \\
\hline \multicolumn{4}{|l|}{ Race, N (\%) } \\
\hline White & $7,242(40.8)$ & $3,436(39.2)$ & $3,806(42.3)$ \\
\hline African American & $9,555(53.8)$ & $4,856(55.4)$ & $4,699(52.2)$ \\
\hline Other Race & $966(5.4)$ & $472(5.4)$ & $494(5.5)$ \\
\hline \multicolumn{4}{|l|}{ Cause of ESRD, $N(\%)$} \\
\hline Diabetes & $7,629(42.9)$ & $4,233(48.3)$ & $3,396(37.7)$ \\
\hline Hypertension & $5,612(31.6)$ & $2,458(28.0)$ & $3,154(35.0)$ \\
\hline Glomerulonephritis & $2,236(12.6)$ & $1,067(12.2)$ & $1,169(13.0)$ \\
\hline Other & $2,286(12.9)$ & $1,006(11.5)$ & $1,280(14.2)$ \\
\hline Body Mass Index, mean (SD), kg/m² & $28.0(7.3)$ & $28.7(7.8)$ & $27.4(6.7)$ \\
\hline \multicolumn{4}{|l|}{ Financial Considerations } \\
\hline Medicaid, N (\%) & $12,206(68.7)$ & $6,351(72.5)$ & $5,855(65.1)$ \\
\hline Low-income subsidy, N (\%) & $14,906(83.9)$ & $7,515(85.7)$ & $7,391(82.1)$ \\
\hline Concomitant medications, $N(\%)^{c}$ & $4.7(3.6)$ & $5.1(3.7)$ & $4.4(3.5)$ \\
\hline \multicolumn{4}{|l|}{ Biochemical Values } \\
\hline Albumin, mean (SD), g/dL ${ }^{d}$ & $3.9(0.4)$ & $3.8(0.4)$ & $4.0(0.4)$ \\
\hline Calcium, mean (SD), mg/dL ${ }^{d}$ & $9.4(0.7)$ & $9.4(0.7)$ & $9.4(0.7)$ \\
\hline Phosphorus, mean (SD), mg/dL ${ }^{d}$ & $5.9(1.7)$ & $5.8(1.7)$ & $6.0(1.7)$ \\
\hline Parathyroid hormone, mean (SD), pg/mL ${ }^{d}$ & $642(519)$ & $640(519)$ & $644(520)$ \\
\hline \multicolumn{4}{|l|}{ Comorbidities } \\
\hline Congestive heart failure, $N(\%)$ & $4,823(27.2)$ & 2,592 (29.6) & 2,231 (24.8) \\
\hline Coronary artery disease/atherosclerosis, $N(\%)$ & $4,703(26.5)$ & $2,434(27.8)$ & $2,269(25.2)$ \\
\hline Cerebrovascular disease, $N(\%)$ & $1,891(10.6)$ & $1,087(12.4)$ & $804(8.9)$ \\
\hline Hypertension, N (\%) & $12,393(69.8)$ & $6,481(74.0)$ & $5,912(65.7)$ \\
\hline Peripheral vascular disease, $N(\%)$ & 2,352 (13.2) & $1,208(13.8)$ & $1,144(12.7)$ \\
\hline Hyperlipidemia, $N(\%)$ & $4,658(26.2)$ & $2,519(28.7)$ & $2,139(23.8)$ \\
\hline Chronic obstructive pulmonary disease or asthma, $N(\%)$ & $2,727(15.4)$ & $1,530(17.5)$ & $1,197(13.3)$ \\
\hline Diabetes, N (\%) & 10,052 (56.6) & $5,491(62.7)$ & $4,561(50.7)$ \\
\hline \multicolumn{4}{|l|}{ Dialysis Care } \\
\hline Phosphorus binder drug, $N(\%)^{\mathrm{e}}$ & $14,135(79.6)$ & $7,037(80.3)$ & $7,098(78.9)$ \\
\hline Catheter access, $N(\%)$ & 3,351 (18.9) & $1,994(22.8)$ & $1,357(15.1)$ \\
\hline Mean intravenous vitamin D dosage, micrograms $(S D)^{f}$ & $12.5(10.3)$ & $11.9(9.9)$ & $13.1(10.7)$ \\
\hline
\end{tabular}

Note: Conversion factors for units: Calcium in $\mathrm{mg} / \mathrm{dL}$ to $\mathrm{mmol} / \mathrm{L}, \mathrm{x} 0.2495 ;$ phosphorus in $\mathrm{mg} / \mathrm{dL}$ to $\mathrm{mmol} / \mathrm{L}, \mathrm{x} 0.3229$

${ }^{a}$ Characteristics were identified using information from Medicare Part A or B claims. A characteristic was considered present if at least one inpatient, home health, or skilled nursing facility claim, or at least two outpatient or physician/supplier claims separated by at least 7 days, were identified during the 6-month baseline period. Additional information concerning covariates can be found in Additional file 1: Table S3

${ }^{b}$ Age and time on dialysis are at the time of cinacalcet initiation

${ }^{c}$ Concomitant medications are the number of concomitant medications at the time of cinacalcet initiation

d Laboratory values were those most proximal to the index date during the baseline period

ePhosphate binders included in the analysis: Sevelamer hydrochloride, sevelamer carbonate, lanthanum carbonate, and calcium acetate

${ }^{f}$ Mean intravenous vitamin D dose per person in the last month of the baseline period. Paricalcitol and doxercalciferol doses were converted to calcitriol-

equivalent doses according to the following conversion ratios: 4.6: 1 for paricalcitol: calcitriol and 3.1: 1 for doxercalciferol: calcitriol 


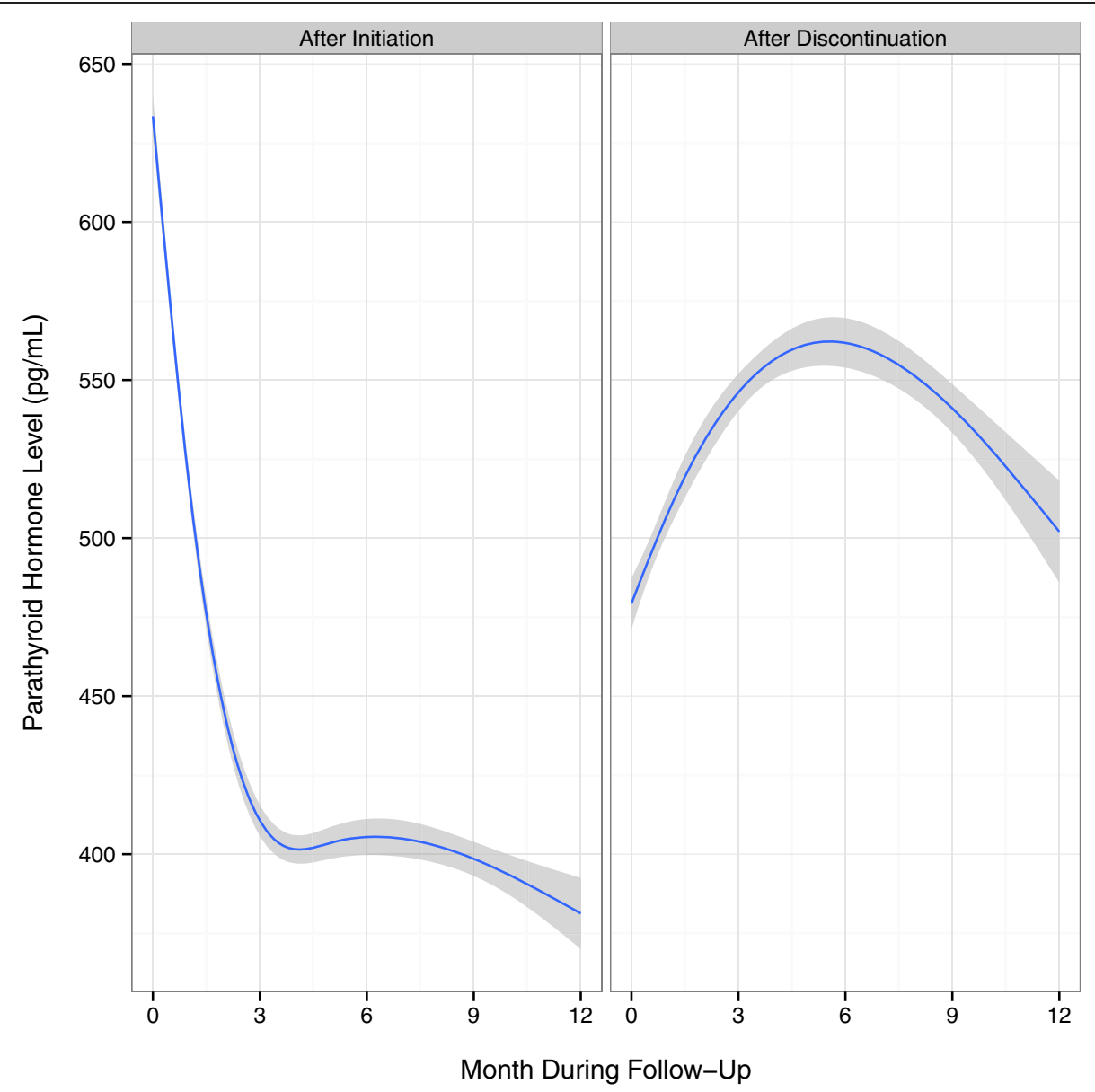

Fig. 1 Mean PTH levels and $95 \%$ confidence intervals by month following cinacalcet initiation and discontinuation

PTH values in all categories examined did not predict reinitiation of cinacalcet. Both increasing and decreasing levels of PTH over time, based on changes in quintile distributions, were both associated with reinitiation of $\mathrm{PTH}$, HR 1.08 (95 \% CI 1.03, 1.14) and HR 1.12 (95\% CI 1.06, 1.19), respectively. Proximal calcium levels both lower and higher were predictive of cinacalcet reinitiation; however, the results of the sensitivity analysis, which used the calcium value 14 days prior to the date of the laboratory value most proximal to reinitiation, showed that only higher calcium levels were associated with reinitiation, HR 1.26 (95 \% CI 1.19, 1.33), Additional file 1: Table S6. All other sensitivity analysis results were not greatly changed when prior laboratory values were used to predict reinitiation. Other predictors of reinitiation included low-income subsidy, HR $=1.32$ (95 \% CI 1.22, 1.43), African American race, $\mathrm{HR}=1.08$ (95\% CI 1.03, 1.13), and higher albumin level, $\mathrm{HR}=1.23$ (95\% CI 1.10, 1.36).

\section{Discussion}

In a cohort of contemporary hemodialysis patients, we observed the effectiveness of cinacalcet in lowering levels of PTH, calcium, and phosphorus, and determined predictors of early cinacalcet discontinuation and subsequent reinitiation. The reductions in these lab values are observed soon after initiation of cinacalcet and mean levels of PTH, calcium, and phosphorus appear to be sustained within recommended target ranges. Yet, discontinuation occurs frequently and relatively soon after initiation of cinacalcet treatment, on average within 4 months following initiation, Additional file 1: Table S7. Furthermore, discontinuation in this population resulted in the loss of biochemical control. Several variables predicted discontinuation. Some of these variables were biochemical, including declining PTH and calcium levels, and others were related to drug prescription drug coverage and novel findings associated with changes in health status. Variables predicting reinitiation were generally similar to those predicting discontinuation.

Average PTH, calcium, and phosphorus levels following cinacalcet initiation in our study were consistent with the 2009 KDIGO guideline update, despite a portion of the follow-up period occurring prior to the release of the guidelines [4]. These guidelines suggest a PTH level in the range of approximately 2 to 9 times the upper reference 


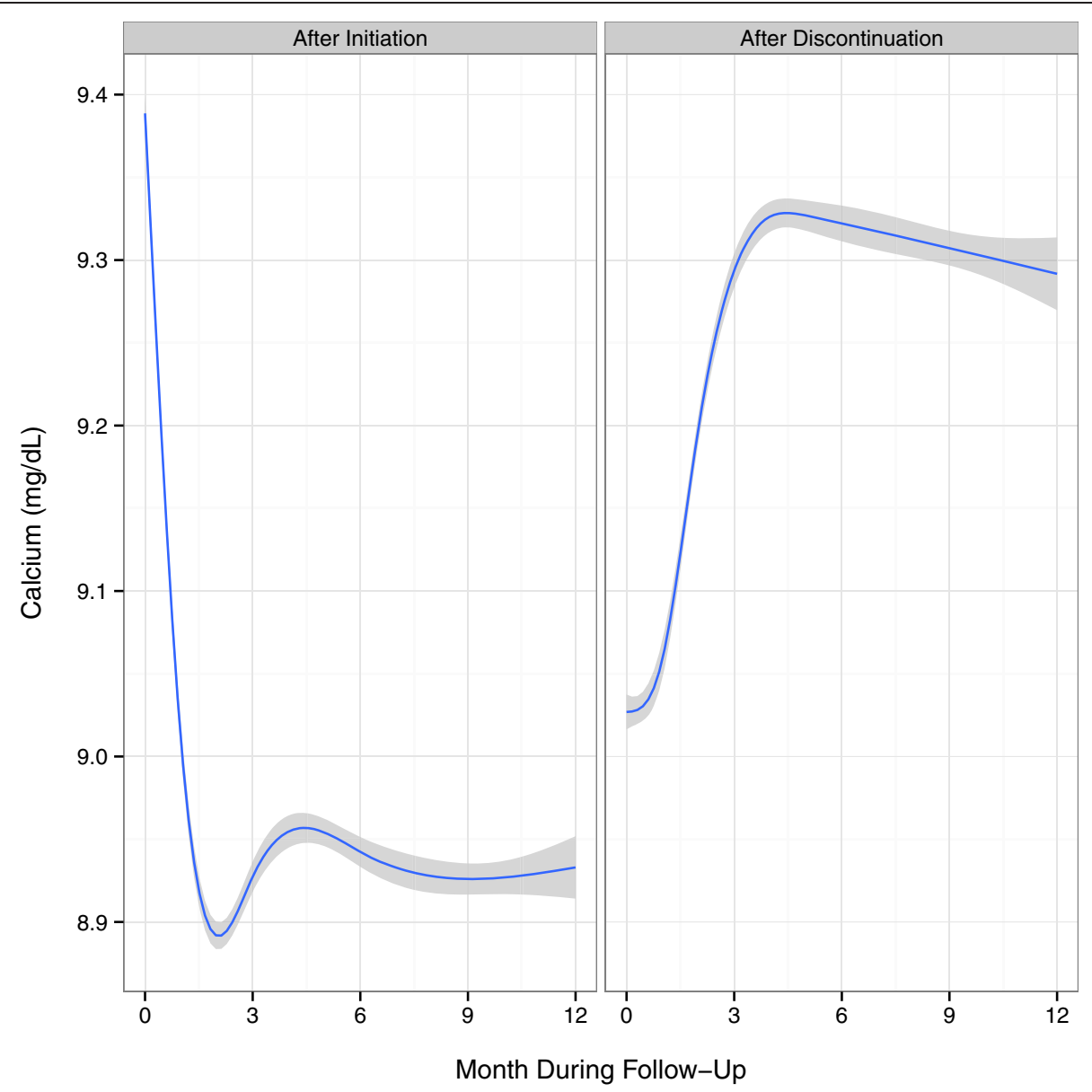

Fig. 2 Mean calcium levels and $95 \%$ confidence intervals by month following cinacalcet initiation and discontinuation. Note: Conversion factors for units: Calcium in $\mathrm{mg} / \mathrm{dL}$ to $\mathrm{mmol} / \mathrm{L}, \times 0.2495$

limit for the assay, which corresponds to approximately 130 to $600 \mathrm{pg} / \mathrm{mL}$, taking into account variability in commercial assays. In our study, average serum calcium levels were maintained in the reference range, in which the recommended upper level of the range is $10.0 \mathrm{mg} / \mathrm{dL}$ to $10.5 \mathrm{mg} / \mathrm{dL}$, depending on the assay used. Average phosphorus levels in our study decreased toward the reference range, which is 2.4 to $4.1 \mathrm{mg} / \mathrm{dL}$, differing slightly among assays. For patients who remained on cinacalcet, biochemical control persisted for up to one year of follow-up. Our results are also similar to those from a recent retrospective cohort study that showed decreases in all three biochemistries that were sustained for 1-year following cinacalcet initiation, although we did not assess dose-titration and its impact on control [7]. Following discontinuation of cinacalcet in the current study, biochemical values increased quickly then leveled off or decreased slightly, Figs. 1, 2 and 3. The slight decreases in biochemical levels are likely due to a selection effect that would result from patients with higher levels restarting treatment and thus being censored from the analysis. Similar to a prior study [16], vitamin D doses in our population decreased following cinacalcet initiation and increased following cinacalcet discontinuation, Additional file 1: Figure S4.

Despite evidence of improved control of SHPT-related biochemical parameters, discontinuation of cinacalcet was common, with $56 \%$ discontinuing by month 4 and $73 \%$ discontinuing by month 12 . This rate of discontinuation is greater than what has been reported in previous cinacalcet studies. Although we cannot know the precise cause of discontinuation, our study is the first to include a large sample size and verify use through Medicare Part D prescription claims, which overcomes many of the possible inaccuracies in start and stop dates [7,10-12]. Prior studies of medication adherence in the general and CKD populations have found that $>50 \%$ of patients demonstrate poor adherence to chronic medications [17]. While poor patient adherence is possible in our population, reinitiation in our population was common and on average occurred 4 months after the first discontinuation. These findings suggest that discontinuation is due to several factors and likely to encompass both patient-initiated (non-adherence) and 


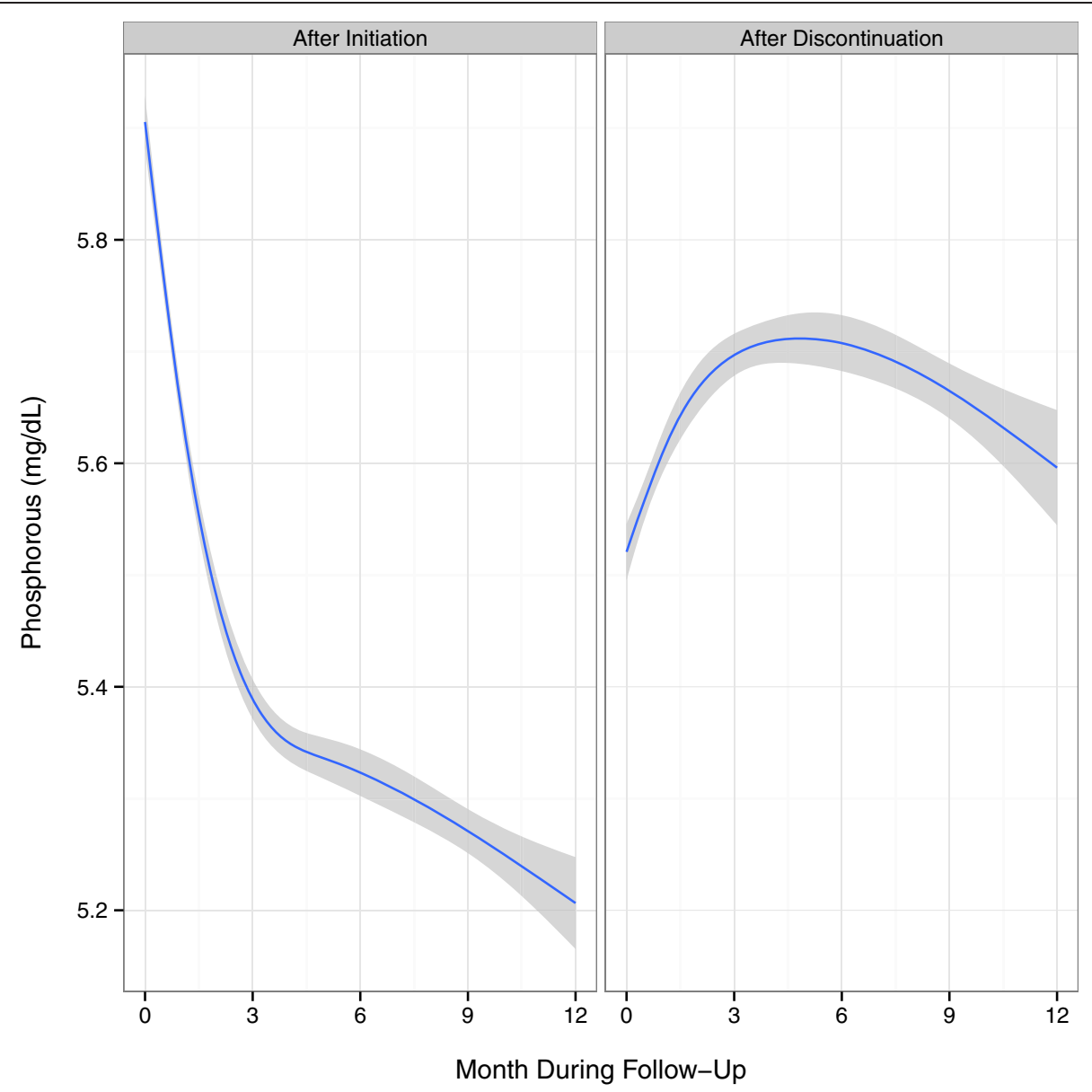

Fig. 3 Mean phosphorus levels and $95 \%$ confidence intervals by month following cinacalcet initiation and discontinuation. Note: Conversion factors for units: phosphorus in $\mathrm{mg} / \mathrm{dL}$ to $\mathrm{mmol} / \mathrm{L}, \times 0.3229$

physician-initiated, medically driven (biochemical value influences) factors.

Our study identified possible predictors that may illuminate why certain patients are discontinued from cinacalcet. While the prescribing information for cinacalcet indicates that administration should be withheld if serum calcium falls below $7.5 \mathrm{mg} / \mathrm{dL}$, only a small proportion of patients experienced hypocalcemia and the modeling revealed it as a weak predictor of discontinuation. PTH levels falling below $150 \mathrm{pg} / \mathrm{mL}$ was more common and was predictive of discontinuation, consistent with prescribing information. Higher doses of cinacalcet at the time of discontinuation also predicted discontinuation, which may further illustrate the role of low or at goal laboratory values in the decision to discontinue cinacalcet. Other possible cinacalcet side effects, nausea, vomiting, and diarrhea [18], were also not common in our study, but did have a weak association with discontinuation. However, because nausea and vomiting are likely to be under reported, measuring this well-recognized side effect is difficult and definitive conclusions from the observed results cannot be drawn for this variable.
We also identified factors related to medication cost that were predictive of discontinuation and reinitiation, suggesting that financial issues could play a role in patients' decisions to discontinue and reinitiate treatment. Patients identified as ever having low-income subsidy status, indicating a lower-out-of pocket cost for cinacalcet, were less likely to discontinue and more likely to reinitiate therapy following discontinuation. Entering the Medicare Part D gap period or being in the Medicare Part D gap period, when out-of-pocket costs are significant, increased the risk of discontinuation. Increasing copay during followup also slightly increased the risk of discontinuation. Our results are similar to the findings of a recent study of adherence and persistence of oral medications, defined as $\geq 80 \%$ medication possession ratio and duration of use, among Medicare Part D beneficiaries receiving dialysis [19]. The investigators found patients not receiving the low-income subsidy and patients entering into the coverage gap were more likely to be non-adherent and had less persistent use of cinacalcet [19]. The investigators found similar relationships with other oral medications indicating that the impact 
Table 2 Predictors of discontinuation and reinitiation

\begin{tabular}{|c|c|c|}
\hline Characteristic $^{a}$ & Discontinuation (HR, $95 \% \mathrm{Cl})$ & Reinitiation (HR, $95 \%$ Cl) \\
\hline Number of time intervals for analysis, $N(\%)$ & $100,706(90.7 \%)$ & $78,789(96.0 \%)$ \\
\hline \multicolumn{3}{|l|}{ Demographics } \\
\hline \multicolumn{3}{|l|}{ Age, years, reference $46-55$} \\
\hline$\leq 45$ & $0.92(0.85,1.00)$ & $0.95(0.90,1.02)$ \\
\hline $56-65$ & $1.05(0.97,1.13)$ & $0.98(0.92,1.04)$ \\
\hline $66-75$ & $1.05(0.97,1.15)$ & $0.90(0.85,0.97)$ \\
\hline$\geq 75$ & $0.98(0.88,1.09)$ & $0.95(0.87,1.04)$ \\
\hline \multicolumn{3}{|l|}{ Time on dialysis, years, reference $<1$} \\
\hline $1-3$ & $1.15(1.01,1.30)$ & $1.00(0.91,1.11)$ \\
\hline$\geq 4$ & $1.14(1.00,1.30)$ & $1.03(0.92,1.14)$ \\
\hline Female & $1.07(1.01,1.13)$ & $1.00(0.96,1.05)$ \\
\hline African American & $1.04(0.98,1.10)$ & $1.08(1.03,1.13)$ \\
\hline \multicolumn{3}{|l|}{ Cause of ESRD, reference diabetes mellitus } \\
\hline Hypertension & $1.02(0.94,1.10)$ & $1.04(0.98,1.11)$ \\
\hline Glomerulonephritis & $1.01(0.90,1.12)$ & $1.08(0.99,1.17)$ \\
\hline Other & $0.96(0.87,1.06)$ & $1.03(0.95,1.12)$ \\
\hline \multicolumn{3}{|l|}{ Body mass index, $\mathrm{kg} / \mathrm{m}^{2}$, reference normal } \\
\hline Underweight & $1.07(0.93,1.23)$ & $0.98(0.87,1.10)$ \\
\hline Overweight & $1.03(0.96,1.10)$ & $1.03(0.98,1.09)$ \\
\hline Obese & $0.98(0.91,1.04)$ & $1.11(1.05,1.17)$ \\
\hline \multicolumn{3}{|l|}{ Financial considerations } \\
\hline Medicaid & $1.03(0.96,1.11)$ & $0.96(0.91,1.02)$ \\
\hline Low-income subsidy & $0.76(0.68,0.85)$ & $1.32(1.22,1.43)$ \\
\hline Concomitant medications in baseline period ${ }^{\mathrm{b}}$ & $0.98(0.97,0.99)$ & $1.00(0.99,1.01)$ \\
\hline Concomitant medications in follow-up period ${ }^{b}$ & $0.96(0.95,0.97)$ & $0.98(0.97,0.99)$ \\
\hline Copay in follow-up period ${ }^{c}$ & $1.04(1.01,1.06)$ & $1.04(1.02,1.06)$ \\
\hline \multicolumn{3}{|l|}{ Last benefit phase in follow-up, reference: covered $^{d}$} \\
\hline Entering the gap period & $1.19(1.00,1.41)$ & $1.01(0.85,1.21)$ \\
\hline Exiting or going through gap period & $0.98(0.77,1.23)$ & $1.03(0.81,1.32)$ \\
\hline In the gap period & $1.10(1.04,1.16)$ & $1.01(0.96,1.06)$ \\
\hline \multicolumn{3}{|l|}{ Biochemical values } \\
\hline \multicolumn{3}{|l|}{ Albumin in baseline period, reference: $<3.3 \mathrm{~g} / \mathrm{dL}$} \\
\hline $3.3-3.9 \mathrm{~g} / \mathrm{dL}$ & $1.11(0.97,1.28)$ & $1.13(1.00,1.27)$ \\
\hline$>3.9 \mathrm{~g} / \mathrm{dL}$ & $1.05(0.91,1.21)$ & $1.09(0.96,1.23)$ \\
\hline \multicolumn{3}{|l|}{ Albumin in follow-up period, reference: $<3.3 \mathrm{~g} / \mathrm{dL}$} \\
\hline $3.3-3.9 \mathrm{~g} / \mathrm{dL}$ & $0.85(0.76,0.95)$ & $1.13(1.02,1.25)$ \\
\hline$>3.9 \mathrm{~g} / \mathrm{dL}$ & $0.78(0.69,0.88)$ & $1.23(1.10,1.36)$ \\
\hline Phosphorus in baseline period, $\mathrm{mg} / \mathrm{dL}$ & $1.02(1.00,1.04)$ & $0.98(0.96,0.99)$ \\
\hline Phosphorus in follow-up period, $\mathrm{mg} / \mathrm{dL}$ & $1.02(1.00,1.04)$ & $0.99(0.98,1.01)$ \\
\hline Parathyroid hormone in baseline period, $\mathrm{pg} / \mathrm{mL}^{\mathrm{e}}$ & $1.00(0.99,1.01)$ & $1.00(0.99,1.00)$ \\
\hline \multicolumn{3}{|c|}{ Parathyroid hormone in follow-up period, reference: $>600 \mathrm{pg} / \mathrm{mL}$} \\
\hline$<150 \mathrm{pg} / \mathrm{mL}$ & $1.24(1.12,1.37)$ & $0.70(0.64,0.76)$ \\
\hline $150-300 \mathrm{pg} / \mathrm{mL}$ & $0.91(0.83,0.99)$ & $0.71(0.66,0.75)$ \\
\hline $301-600 \mathrm{pg} / \mathrm{mL}$ & $0.89(0.82,0.97)$ & $0.85(0.80,0.90)$ \\
\hline
\end{tabular}


Table 2 Predictors of discontinuation and reinitiation (Continued)

\begin{tabular}{|c|c|c|}
\hline \multicolumn{3}{|l|}{ Parathyroid hormone in follow-up period, change in quintiles, reference: no change ${ }^{f}$} \\
\hline Increase & $1.15(1.07,1.23)$ & $1.08(1.03,1.14)$ \\
\hline Decrease & $0.90(0.84,0.97)$ & $1.12(1.06,1.19)$ \\
\hline Calcium in baseline period, $\mathrm{mg} / \mathrm{dL}$ & $0.95(0.91,0.99)$ & $1.16(1.12,1.20)$ \\
\hline \multicolumn{3}{|l|}{ Calcium in follow-up period, reference: $>8.7 \mathrm{mg} / \mathrm{dL}^{\mathrm{g}}$} \\
\hline$<7.5 \mathrm{mg} / \mathrm{dL}$ & $1.07(0.89,1.29)$ & $1.12(0.91,1.39)$ \\
\hline 7.5-8.7 mg/dL & $0.85(0.80,0.91)$ & $1.26(1.19,1.33)$ \\
\hline \multicolumn{3}{|l|}{ Calcium in follow-up period, change in quintiles, reference: no change ${ }^{f}$} \\
\hline Increase & $1.24(1.16,1.32)$ & $1.07(1.02,1.13)$ \\
\hline Decrease & $0.94(0.88,1.00)$ & $1.04(0.99,1.10)$ \\
\hline \multicolumn{3}{|l|}{ Comorbidities } \\
\hline Congestive heart failure in baseline period & $1.06(0.99,1.13)$ & $0.96(0.91,1.01)$ \\
\hline Congestive heart failure in follow-up period & $1.01(0.90,1.14)$ & $1.11(0.94,1.31)$ \\
\hline Coronary artery disease/atherosclerosis in baseline period & $1.01(0.95,1.09)$ & $0.93(0.88,0.99)$ \\
\hline Cerebrovascular disease in baseline period & $0.94(0.86,1.02)$ & $1.00(0.93,1.07)$ \\
\hline Stroke in follow-up period & $1.30(1.05,1.60)$ & $0.82(0.55,1.20)$ \\
\hline Hypertension in baseline period & $1.12(1.05,1.19)$ & $1.03(0.98,1.08)$ \\
\hline Peripheral vascular disease in baseline period & $1.02(0.94,1.11)$ & $1.11(1.03,1.19)$ \\
\hline Peripheral vascular disease in follow-up period & $0.99(0.85,1.16)$ & $0.91(0.76,1.09)$ \\
\hline Hyperlipidemia in baseline period & $1.01(0.95,1.07)$ & $1.06(1.01,1.12)$ \\
\hline Chronic obstructive pulmonary disease and asthma in baseline period & $1.00(0.93,1.08)$ & $1.00(0.94,1.06)$ \\
\hline Diabetes in baseline period & $1.05(0.98,1.14)$ & $0.98(0.92,1.04)$ \\
\hline Nausea, vomiting, diarrhea in follow-up period & $1.09(0.91,1.32)$ & $1.05(0.85,1.31)$ \\
\hline Seizure in follow-up period & $1.18(0.93,1.49)$ & $1.11(0.82,1.50)$ \\
\hline \multicolumn{3}{|l|}{ Dialysis care } \\
\hline Intravenous vitamin D in baseline period ${ }^{\text {h }}$ & $1.01(0.98,1.05)$ & $0.98(0.96,1.00)$ \\
\hline Intravenous vitamin D in follow-up period ${ }^{\text {h }}$ & $0.94(0.91,0.97)$ & $1.02(0.99,1.04)$ \\
\hline Phosphorus binder drug in baseline period ${ }^{i}$ & $1.02(0.95,1.10)$ & $1.12(1.06,1.18)$ \\
\hline Phosphorus binder drug in follow-up period ${ }^{i}$ & $0.77(0.73,0.82)$ & $1.03(0.98,1.08)$ \\
\hline Catheter access in baseline period & $0.97(0.89,1.07)$ & $1.07(0.99,1.15)$ \\
\hline Catheter access in follow-up period & $1.10(1.00,1.21)$ & $0.83(0.77,0.90)$ \\
\hline \multicolumn{3}{|l|}{ Most recent dose of cinacalcet, reference: 30 mg } \\
\hline $60 \mathrm{mg}$ & $1.07(1.00,1.15)$ & $\mathrm{n} / \mathrm{a}$ \\
\hline $90 \mathrm{mg}$ & $1.15(1.03,1.29)$ & $\mathrm{n} / \mathrm{a}$ \\
\hline \multicolumn{3}{|l|}{ Days in the hospital in follow-up period, reference: 0 days } \\
\hline 1-4 days & $2.02(1.84,2.22)$ & $0.85(0.75,0.96)$ \\
\hline$\geq 5$ days & $1.90(1.73,2.08)$ & $0.79(0.69,0.89)$ \\
\hline
\end{tabular}

Note: Conversion factors for units: Calcium in $\mathrm{mg} / \mathrm{dL}$ to $\mathrm{mmol} / \mathrm{L}, \mathrm{x} 0.2495$; phosphorus in $\mathrm{mg} / \mathrm{dL}$ to $\mathrm{mmol} / \mathrm{L}, \times 0.3229$

a Baseline characteristics were identified using information from Medicare Part A or B claims. A characteristic was considered present if at least one inpatient, home health, or skilled nursing facility claim, or at least two outpatient or physician/supplier claims separated by at least 7 days, were identified during the 6-month baseline period. Additional information concerning baseline characteristics can be found in Additional file 1: Table S4. Time-varying (follow-up) characteristics were evaluated at 30-day intervals following the start of follow-up. Additional information concerning time-varying (follow-up) characteristics can be found in Additional file 1: Table S2

${ }^{\mathrm{b}}$ Concomitant medications are the number of concomitant medications at the time of cinacalcet discontinuation or reinitiation

${ }^{\circ}$ Changes in co-pay were based on increments of $\$ 100$. The last co-pay prior to discontinuation was used to predict cinacalcet reinitiation

${ }^{d}$ Benefit phase reflects the status of Medicare Part $D$ coverage at the time of the fill of cinacalcet

${ }^{e}$ Changes in parathyroid hormone level were based on increments of $100 \mathrm{pg} / \mathrm{mL}$

fDistributions of parathyroid hormone and calcium were examined across all of follow-up and quintiles were based on these distributions. Increase indicates an increase to another quintile and trend of increasing laboratory levels. Decrease indicates a decrease to another quintile and a trend of decreasing laboratory levels 


\begin{abstract}
${ }^{9}$ Results presented for prediction of reinitiation associated with follow-up calcium levels are those from the sensitivity analysis utilizing a lag time of 14 days. The calcium level recorded 14 days prior to the date of the laboratory value most proximal to discontinuation was used to predict reinitiation. All other results were not significantly changed when lag times were considered

${ }^{\mathrm{h}}$ Mean intravenous vitamin D dose was assessed in the last month of the baseline period. Changes in intravenous vitamin D dose were based in increments of $10 \mathrm{mcg}$. Paricalcitol and doxercalciferol doses were converted to calcitriol-equivalent doses according to the following conversion ratios: $4.6: 1$ for paricalcitol: calcitriol and 3.1: 1 for doxercalciferol: calcitriol

'Phosphate binders included in the analysis: Sevelamer hydrochloride, sevelamer carbonate, lanthanum carbonate, and calcium acetate
\end{abstract}

of economic burden in this population is not specific to cinacalcet. Therapy regimens for patients receiving dialysis are burdensome, and patients are prescribed on average 10-12 tablets per day, all likely leading to financial burden [10, 20, 21].

It has been recommended that therapeutic decisions in CKD patients be based on trends, rather than single laboratory values [4]. Therefore, in addition to examining the laboratory values most proximal to the time of discontinuation, we also examined the changes in lab values leading up to discontinuation. Our most striking finding was that trends of increasing levels of PTH and calcium over time were associated with discontinuation. This finding could be an early signal of non-adherence. As patients become non-adherent to cinacalcet due to side effects, financial issues, or medication complexity, rising levels of PTH and calcium could be apparent and a signal to clinicians to consider that factors other than medication ineffectiveness may be an issue. Identifying ways to decrease medication complexity, financial burdens, and side effects could enable patients to persist longer on cinacalcet therapy.

Several limitations of our study must be noted. First, medication use information was obtained from pharmacy claims, which are an imperfect measure of actual medication consumed. It is possible that patients were obtaining medications outside of their Medicare Part D benefit $[22,23]$. We also cannot precisely determine the day when patients stops or reinitiates treatment. However, we adopted a design to minimize the likelihood that predictors would be assessed following discontinuation or reinitiation events, thus avoiding the problem of predictors being consequences of discontinuation or reinitiation, rather than causes. In addition, we performed a sensitivity analysis to determine if a delay in physician recognition and response to a laboratory value could affect reinitiation results. Adding a lag in the dates of the biochemical values affected calcium reinitiation results, a biochemical test drawn frequently, but not PTH results, a biochemical test drawn less frequently. In future studies using claims data, consideration of the time frame of when frequent biochemical values are drawn and when a physician would realistically identify and act on a biochemical value should be considered. Finally, many of our variables were assessed using ICD9 codes associated with health care encounters. Many of these definitions, such as those for acute myocardial infarction [24], are known to have very high sensitivity and specificity, but others, such as those for nausea, are likely to be much less sensitive.

\section{Conclusions}

The results of our study indicate that on average biochemical control of PTH, calcium, and phosphorus following cinacalcet initiation were consistent with recommendations in the 2009 KDIGO guideline update. Yet, early discontinuation of cinacalcet was frequent and resulted in the loss of control of PTH, calcium, and phosphorus. Both economic and clinical factors contribute to cinacalcet discontinuation, as well as reinitiation. Examining trends in laboratory values over time could identify early signals of non-adherence and present an opportunity for clinicians to intervene. In conclusion, prolonged biochemical control was achieved by continued cinacalcet therapy and factors associated with discontinuation and reinitiation indicate persistent use of and adherence to cinacalcet are impacted by a range of both modifiable and immutable factors.

\section{Additional file}

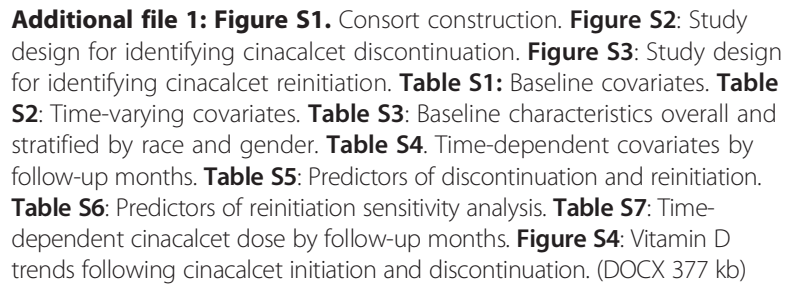

Additional file 1: Figure S1. Consort construction. Figure S2: Study design for identifying cinacalcet discontinuation. Figure S3: Study design for identifying cinacalcet reinitiation. Table S1: Baseline covariates. Table S2: Time-varying covariates. Table S3: Baseline characteristics overall and stratified by race and gender. Table S4. Time-dependent covariates by follow-up months. Table S5: Predictors of discontinuation and reinitiation. Table S6: Predictors of reinitiation sensitivity analysis. Table S7: Timedependent cinacalcet dose by follow-up months. Figure S4: Vitamin D trends following cinacalcet initiation and discontinuation. (DOCX 377 kb)

\section{Abbreviations}

SHPT: Secondary hyperparathyroidism; CKD: Chronic kidney disease; PTH: Parathyroid hormone; CKD-MBD: Chronic kidney disease-mineral and bone disorder; KDIGO: Kidney Disease: Improving Global Outcomes; USRDS: United States Renal Data System; ESRD: End-stage renal disease; SD: Standard deviation; HR: Hazard ratio; ICD-9: International Classification of Diseases Ninth Revision codes; CPT: Current Procedural Terminology codes; HCPCS: Healthcare Common Procedure Coding System codes.

\section{Competing interests}

Some of the data reported here have been supplied by the United States Renal Data System (USRDS). The interpretation and reporting of these data are the responsibility of the authors and in no way should be seen as an official policy or interpretation of the U.S. government or the USRDS. The authors thank DaVita Clinical Research for providing data for this study. DaVita Clinical Research had no role in the design or implementation of this study, nor on the decision to publish.

Conflict of interest disclosures: M.A.B. has received investigator-initiated grant support from Amgen and served as a scientific advisor for Pfizer, Merck, and Amgen, (compensation received by institution). M.A.B. has received consulting fees from RxAnte, and World Health Information Consultants for unrelated work. B.D.R and A.V.K. have received investigator-initiated grant support from Amgen, Inc. A.V.K. served on a Fresenius Advisory Board. 


\section{Authors' contributions}

$M A B, P J D$, and BDB conceived the study concept and design. MAB and BDR acquired the data. All authors participated in the analysis and interpretation of the results. BDR and MAB drafted the manuscript. All authors participated in the critical revision of the manuscript for important intellectual content. $M A B$ performed the statistical analysis and obtained funding. BDR and MAB provided administrative, technical, and material support. MAB supervised the study and takes responsibility that this study has been reported honestly, accurately, and transparently; and that no important aspects of the study have been omitted. All authors read and approved the final manuscript.

\section{Acknowledgements}

The authors thank DaVita Clinical Research and the USRDS for providing data for this study. DaVita Clinical Research and the USRDS had no role in the design or implementation of this study or in the decision to publish. In addition, the opinions expressed are those of the authors and not of DaVita, the NIDDK or USRDS.

This study was supported by a research contract with Amgen, Inc, Thousand Oaks, CA. Before submission for peer review, the sponsor reviewed the manuscript. Comments were sent to the authors, who are solely responsible for the final version. The analysis, interpretation, and reporting of these data are the responsibility of the authors.

\section{Author details}

${ }^{1}$ Cecil G. Sheps Center for Health Services Research, University of North Carolina, Chapel Hill, NC, USA. ${ }^{2}$ Amgen, Inc, Thousand Oaks, CA, USA. ${ }^{3}$ University of North Carolina Kidney Center, UNC School of Medicine, Chapel Hill, NC, USA. ${ }^{4}$ Department of Epidemiology, UNC Gillings School of Global Public Health, UNC Chapel Hill, Chapel Hill, NC, USA.

Received: 26 May 2015 Accepted: 19 October 2015

\section{Published online: 29 October 2015}

\section{References}

1. Cunningham J. Management of secondary hyperparathyroidism. Ther Apher Dial. 2005;9 Suppl 1:S35-40

2. Goodman WG, Quarles LD. Development and progression of secondary hyperparathyroidism in chronic kidney disease: lessons from molecular genetics. Kidney Int. 2008;74(3):276-88.

3. Cunningham J, Locatelli F, Rodriguez M. Secondary hyperparathyroidism: pathogenesis, disease progression, and therapeutic options. Clin J Am Soc Nephrol. 2011;6(4):913-21.

4. CKDMBDWG, KDIGO. KDIGO clinical practice guideline for the diagnosis, evaluation, prevention, and treatment of Chronic Kidney Disease-Mineral Bone Disorder (CKD-MBD). Kidney Int Suppl. 2009;113:S1-130.

5. Block GA, Martin KJ, de Francisco AL, Turner SA, Avram MM, Suranyi MG, et al. Cinacalcet for secondary hyperparathyroidism in patients receiving hemodialysis. N Engl J Med. 2004;350(15):1516-25.

6. Lindberg JS, Culleton B, Wong G, Borah MF, Clark RV, Shapiro WB, et al. Cinacalcet $\mathrm{HCl}$, an oral calcimimetic agent for the treatment of secondary hyperparathyroidism in hemodialysis and peritoneal dialysis: a randomized double-blind, multicenter study. J Am Soc Nephrol. 2005;16(3):800-7.

7. Kilpatrick RD, Newsome BB, Zaun D, Liu J, Solid CA, Nieman K, et al. Evaluating real-world use of cinacalcet and biochemical response to therapy in US hemodialysis patients. Am J Nephrol. 2013:37(4):389-98.

8. Smrzova J, Urbanek T. Cinacalcet - clinical and laboratory effectiveness, concomitant treatment patterns and treatment cost: could we do bette and how? Kidney Blood Press Res. 2010;33(5):333-42.

9. Urena $\mathrm{P}$, Jacobson SH, Zitt E, Vervloet M, Malberti F, Ashman N, et al, Cinacalcet and achievement of the NKF/K-DOQI recommended target values for bone and mineral metabolism in real-world clinical practice-the ECHO observational study. Nephrol Dial Transplant. 2009;24(9):2852-9.

10. Gincherman Y, Moloney K, McKee C, Coyne DW. Assessment of adherence to cinacalcet by prescription refill rates in hemodialysis patients. Hemodial Int. 2010;14(1):68-72

11. Lee A, Song X, Khan I, Belozeroff V, Goodman W, Fulcher N, et al. Association of cinacalcet adherence and costs in patients on dialysis. J Med Econ. 2011:14(6):798-804

12. Pruijm M, Teta D, Halabi G, Wuerzner G, Santschi V, Burnier M. Improvement in secondary hyperparathyroidism due to drug adherence monitoring in dialysis patients. Clin Nephrol. 2009;72(3):199-205.
13. Strippoli GF, Palmer S, Tong A, Elder G, Messa P, Craig JC. Meta-analysis of biochemical and patient-level effects of calcimimetic therapy. Am J Kidney Dis. 2006;47(5):715-26

14. Ray WA. Evaluating medication effects outside of clinical trials: new-user designs. Am J Epidemiol. 2003;158(9):915-20.

15. R: A Language and Environment for Statistical Computing. In. Vienna, Austria: R Foundation for Statistical Computing; 2014.

16. Newsome BB, Kilpatrick RD, Liu J, Zaun D, Solid CA, Nieman K, et al. Racial differences in clinical use of cinacalcet in a large population of hemodialysis patients. Am J Nephrol. 2013;38(2):104-14.

17. Schmid $H$, Hartmann B, Schiff $H$. Adherence to prescribed oral medication in adult patients undergoing chronic hemodialysis: a critical review of the literature. Eur J Med Res. 2009:14(5):185-90.

18. Investigators ET, Chertow GM, Block GA, Correa-Rotter R, Drueke TB, Floege $J$, et al. Effect of cinacalcet on cardiovascular disease in patients undergoing dialysis. N Engl J Med. 2012;367(26):2482-94.

19. Park H, Rascati KL, Lawson KA, Barner JC, Richards KM, Malone DC Adherence and persistence to prescribed medication therapy among Medicare part $D$ beneficiaries on dialysis: comparisons of benefit type and benefit phase. J Manag Care Pharm. 2014;20(8):862-76.

20. Chiu YW, Teitelbaum I, Misra M, de Leon EM, Adzize T, Mehrotra R. Pill burden, adherence, hyperphosphatemia, and quality of life in maintenance dialysis patients. Clin J Am Soc Nephrol. 2009;4(6):1089-96.

21. Manley HJ, Garvin CG, Drayer DK, Reid GM, Bender WL, Neufeld TK, et al. Medication prescribing patterns in ambulatory haemodialysis patients: comparisons of USRDS to a large not-for-profit dialysis provider. Nephro Dial Transplant. 2004;19(7):1842-8

22. Li X, Sturmer T, Brookhart MA. Evidence of sample Use among New users of statins: implications for pharmacoepidemiology. Med Care. 2014.

23. Lauffenburger JC, Balasubramanian A, Farley JF, Critchlow CW, O'Malley CD, Roth MT, et al. Completeness of prescription information in US commercial claims databases. Pharmacoepidemiol Drug Saf. 2013;22(8):899-906.

24. Brouwer ES, Napravnik S, Eron Jr JJ, Simpson Jr RJ, Brookhart MA, Stalzer B, et al. Validation of medicaid claims-based diagnosis of myocardial infarction using an HIV clinical cohort. Med Care. 2013.

\section{Submit your next manuscript to BioMed Central and take full advantage of:}

- Convenient online submission

- Thorough peer review

- No space constraints or color figure charges

- Immediate publication on acceptance

- Inclusion in PubMed, CAS, Scopus and Google Scholar

- Research which is freely available for redistribution 